\title{
HEMISELMIS ANDERSENII AND CHLORELLA STIGMATOPHORA AS NEW SOURCES OF HIGH-VALUE COMPOUNDS: A LIPIDOMIC APPROACH ${ }^{1}$
}

\author{
Tomásia Fernandes (D) and Nereida Cordeiro (iD ${ }^{2}$ \\ Faculty of Sciences and Engineering, University of Madeira, Campus Universitário da Penteada, 9020-105 Funchal, Portugal \\ CIIMAR-Interdisciplinary Centre of Marine and Environmental Research, University of Porto, 4450-208 Matosinhos, Portugal
}

To unlock the potential of Chlorella stigmatophora (Trebouxiophyceae, Chlorophyta) and Hemiselmis andersenii (Cryptophyceae, Cryptophyta) as natural reactors for biotechnological exploitation, their lipophilic extracts were characterized using Fourier Transform Infrared spectroscopy with Attenuated Total Reflectance (FTIR-ATR) and Gas ChromatographyMass Spectrometry (GC-MS) before and after alkaline hydrolysis. The GC-MS analysis enabled the identification of 62 metabolites-namely fatty acids (27), aliphatic alcohols (17), monoglycerides (7), sterols (4), and other compounds (7). After alkaline hydrolysis, monounsaturated fatty acids increased by as much as $87 \%$, suggesting that the esterified compounds were mainly neutral lipids. Hemiselmis andersenii yielded the highest $\Sigma \omega 3 / \Sigma \omega 6$ ratio (7.26), indicating that it is a good source of $\omega 3$ fatty acids, in comparison to C. stigmatophora $(\Sigma \omega 3 / \Sigma \omega 6=1.24)$. Both microalgae presented significant amounts of aliphatic alcohols (6.81-10.95 $\left.\mathrm{mg} \cdot \mathrm{g} \mathrm{dw}^{-1}\right)$, which are recognized by their cholesterol-lowering properties. The multivariate analysis allowed visualization of the chemical divergence among $H$. andersenii lipophilic extracts before and after alkaline hydrolysis, as well as speciesspecific differences. Chlorella stigmatophora showed to be a valuable source of essential fatty acids for nutraceuticals, whereas $H$. andersenii, due to its high chemical diversity, seems to be suitable for different fields of application.

Key index words: Chlorella stigmatophora; GC-MS; Hemiselmis andersenii; lipophilic fraction; microalgae

Abbreviations: $\mu$, growth rate; AA, aliphatic alcohols; $\mathrm{AH}$, after alkaline hydrolysis; $\mathrm{BH}$, before alkaline hydrolysis; DHA, docosahexaenoic acid (C22:6ஸ3); EPA, eicosapentaenoic acid (C20:5 13$)$; FA, fatty acids; FTIR-ATR, fourier transform infrared spectroscopy with attenuated total reflectance; GC-MS, gas chromatography-mass spectrometry; IS, internal standard; LCAA, long-chain aliphatic alcohols; MG, monoglycerides; MUFA, monounsaturated fatty acids; PCA, principal component analysis; PUFA, polyunsaturated fatty acids; SFA, saturated fatty

\footnotetext{
${ }^{1}$ Received 13 January 2020. Accepted 9 June 2020.

${ }^{2}$ Author for correspondence e-mail ncordeiro@staff.uma.pt.

Editorial Responsibility: Y. Li (Associate Editor)
}

acids; ST, sterols; TMS, trimethylsilyl derivatives; $\Sigma \omega 3 / \Sigma \omega 6$, ratio between the total of omega 3 and omega 6 fatty acids

Microalgae show dual potential as a feedstock for biomass and biofuel production. The advantages that make these organisms sustainable biofactories to synthesize value-added compounds include noncompetition for agricultural land, carbon fixation ability, and metabolic plasticity (Rocha et al. 2017). From the wide biological diversity of microalgae and cyanobacteria, only a few species belonging to Arthrospira and Chlorella genera dominate the human nutrition market, with an estimated global production of 6,600 and 12,000 tons of dry matter per year, respectively (Kent et al. 2015, Muys et al. 2019).

Among algal metabolites, lipids are of great importance for the biotechnological exploitation of microalgae (Matich et al. 2018). For instance, essen-

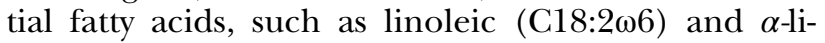
nolenic (C18:3 $\omega 3)$ acids, can be suitable for applications in the food industry as an additive and/ or nutritional supplement, while triacylglycerols and saturated fatty acids are suitable for biofuel production (Matich et al. 2018). Additionally, given the reduction of global fish stocks and fishes' susceptibility to contamination by pollutants (e.g., heavy metals), microalgae have emerged as sustainable sources of long-chain polyunsaturated fatty acids (Guiheneuf and Stengel 2013, Gheysen et al. 2018). Besides fatty acids, microalgae-derived lipids also contain aliphatic alcohols, monoglycerides, and phytosterols which have potential applications in aquaculture feed, food, and pharmaceutical industries (Luo et al., 2015). Indeed, phytosterols have been incorporated in food matrices such as low-fat milk, fat-based spreads, and cereal bars due to their efficacy as cholesterol-lowering agents (Jones and AbuMweis 2009).

Cryptophyceaen species are used for application in aquaculture industries due to their high polyunsaturated fatty acids and sterol concentrations, but are not widely produced for the purpose of human health (Peltomaa et al. 2017). Hemiselmis andersenii belongs to this taxonomic class and research efforts have been focused on its nucleomorph 
characterization to study the process of genome evolution (Lane et al. 2007). The genus Chlorella is recognized as a model oleaginous microalga, Chlorella stigmatophora being one of the few marine species that belongs to this genus (Jiang et al. 2012). In both microalgal species, the analysis of the lipophilic fraction is limited to specific families like fatty acids and sterols (Slocombe et al. 2015). The abundance of other important lipophilic classes, such as long-chain aliphatic alcohols and monoglycerides, is unknown.

Lipidomics play a key role in deciphering the potential of microalgae as natural reactors to produce bioactive lipids (da Costa et al. 2016). This area of metabolomics is responsible for the detailed identification and quantification of the lipid species (da Costa et al. 2016). In this field of study, GC-MS is often applied due to its low sample requirement and because it is a powerful analytical tool that enables the robust characterization of the total lipid extract (da Costa et al. 2016). In this sense, GC-MS techniques have been used for the complete study of the lipid extract as a whole (Santos et al. 2015, Rahmouni et al. 2017).

The main aim of the present work was to evaluate the potential of Hemiselmis andersenii and Chlorella stigmatophora strains as potential sources of highvalue compounds for further applications in nutraceutical and other related fields. With this purpose, the profiles of the lipophilic extracts from C. stigmatophora and $H$. andersenii were analyzed using GC-MS. Since the lipophilic fraction can be enriched in molecules in their esterified forms, the study of complex lipids is crucial. Thus, the characterization of the lipophilic extracts was made before and after alkaline hydrolysis.

\section{MATERIALS AND METHODS}

Growth and culture conditions. Microalgae were obtained from the Roscoff Culture Collection (RCC) and the Spanish Bank of Algae (BEA). The cultivation of the Chlorophyta Chlorella stigmatophora (RCC 661) and the Cryptophyta Hemiselmis cf. andersenii (BEA 0118B) was performed by inoculating starter cultures in sterile $\mathrm{f} / 2-\mathrm{Si}$ medium with $\mathrm{pH}$ adjusted to 7.0 , under $70 \mu \mathrm{mol}$ photons $\cdot \mathrm{m}^{-2} \cdot \mathrm{s}^{-1}$ light intensity and $16: 8 \mathrm{~h}$ light:dark cycles, at $25^{\circ} \mathrm{C}$. At the end of the logarithmic phase, cultures were harvested by centrifugation at $3720 \mathrm{~g}$, and pellets were washed twice with an isotonic saline solution $\left(0.09 \mathrm{~g} \mathrm{NaCl} \cdot \mathrm{L}^{-1}\right)$. Cell densities were determined with a Neubauer-improved counting chamber (Marienfeld Superior) and a light microscope (Olympus BX41, Tokyo, Japan) with $40 \times$ magnification. The specific growth rate was obtained by the linearization of the following logistic model (Equation 1):

$$
N=\frac{K}{1+e^{a-\mu t}}
$$

where $K\left(\right.$ cells $\left.\cdot \mathrm{mL}^{-1}\right)$ stands for the carrying capacity, $N$ (cells $\cdot \mathrm{mL}^{-1}$ ) corresponds to the cell concentration in time $(t), a$ is the position of the origin, and $\mu\left(\mathrm{d}^{-1}\right)$ is the specific growth rate (Xin et al. 2010, Fernandes et al. 2016).
Biomass yield $\left(\mathrm{g} \cdot \mathrm{L}^{-1}\right.$; in dry weight) was estimated at the end of the batch cultivation by freeze-drying algal samples on pre-weighed flasks. Dry weight $(\mathrm{dw})$ was obtained based on weight difference.

Extraction of the lipophilic fraction. The lipophilic phase was extracted as reported in Ma et al. (2015), with some modifications. Approximately 100-150 mg of freeze-dried sample was extracted with an aqueous solution (methanol:water, 1:1) and chloroform in a 1:1 ratio. The mixture was left stirring for $15 \mathrm{~min}$ and separated by centrifugation at $4,427 \mathrm{~g}$ for $10 \mathrm{~min}$. The lipophilic phase was transferred into pre-weighed tubes and evaporated in a nitrogen atmosphere before hydrolysis $(\mathrm{BH})$. The amount of extractable substances was gravimetrically quantified and expressed as a percentage by weight of the freeze-dried biomass $(\mathrm{dw})$.

Fourier transform infrared spectroscopy with attenuated total reflectance (FTIR-ATR). FTIR-ATR spectra of microalgal raw freezedried biomass and lipophilic extracts $\mathrm{BH}$ were collected on a Perkin-Elmer Spectrum Two instrument coupled with a Diamond ATR accessory (DurasamplIR II, Smiths Detection, UK) scanning over the wave number range of $4,000-650 \cdot \mathrm{cm}^{-1}$ at a resolution of $4 \cdot \mathrm{cm}^{-1}$ and 36 scans.

Alkaline hydrolysis and GC-MS analysis. The alkaline hydrolysis was performed in two aliquots according to Santos et al. (2015). To each extract, $10 \mathrm{~mL}$ of $0.5 \mathrm{M} \mathrm{NaOH}$ in $50 \%$ aqueous methanol solution was added and the mixture heated at $100^{\circ} \mathrm{C}$ for $1 \mathrm{~h}$ in a nitrogen atmosphere. Samples were acidified to $\mathrm{pH} 2$ with $1 \mathrm{M} \mathrm{HCl}$ and extracted with dichloromethane. The solvent was evaporated to dryness under nitrogen and named $\mathrm{AH}$.

To fully exploit the potential of the lipophilic fraction of Chlorella stigmatophora and Hemiselmis andersenii, an untargeted approach was implemented. Two aliquots of each dried extract (before- $\mathrm{BH}$ and after- $\mathrm{AH}$ alkaline hydrolysis) were silylated according to the methodology described by Santos et al. (2015). Briefly, an accurate amount of internal standard (IS; tetracosane $0.20-0.30 \mathrm{mg}$ ), $250 \mu \mathrm{L}$ of pyridine, $250 \mu \mathrm{L}$ of $\mathrm{N}, \mathrm{O}$-bis (trimethylsilyl) trifluoroacetamide, and $50 \mu \mathrm{L}$ of trimethylchlorosilane were added to the extracts. The mixtures were then left to react at $70^{\circ} \mathrm{C}$ for $30 \mathrm{~min}$.

The trimethylsilyl (TMS) derivatives were analyzed in a gas chromatograph (Agilent HP 6890, Palo Alto, CA, USA) equipped with a mass selective detector (Agilent 5973) and a ValcoBon 17704 capillary column VB1 $(30 \mathrm{~m} \times 0.25 \mathrm{~mm}$ inner diameter, $0.25 \mu \mathrm{m}$ film thickness). The temperature gradient started with an initial temperature of $80^{\circ} \mathrm{C}$ for $5 \mathrm{~min}$; a linear increase to $208^{\circ} \mathrm{C}$ at $4^{\circ} \mathrm{C} \cdot \mathrm{min}^{-1}$, followed by an increase to reach $260^{\circ} \mathrm{C}$ at $2^{\circ} \mathrm{C} \cdot \mathrm{min}^{-1}$, and a final increase to $300^{\circ} \mathrm{C}$ at $5^{\circ} \mathrm{C} \mathrm{min}^{-1}$ with $4 \mathrm{~min}$ of hold time. The temperature of the injector was $250^{\circ} \mathrm{C}$; the transfer line, $290^{\circ} \mathrm{C}$; and the split ratio was $33: 1$. Helium was used as the

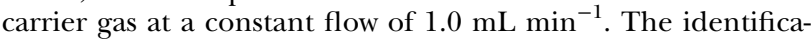
tion of the TMS derivatives was made by comparison of the mass spectra fragmentation to those in the GC-MS spectral library (Wiley-NIST Mass Spectral Library 1999), published data (Khannoon et al. 2011, Christie 2018, Xu et al. 2018) or by injection of standards. For semiquantitative analysis, the GC-MS was calibrated with pure reference compounds (mannose, trans-ferulic acid, nonadecan-1-ol, eicosan-1-ol, $5 \alpha-\mathrm{c}-$ holestane, cholesterol, stigmasterol, hexadecenoic acid, and nonadecanoic acid) and the response factor was calculated toward tetracosane (IS).

Statistical analysis. Statistical analysis of the data was carried out using the software IBM SPSS Statistics 24, IBM Corporation, Armonk, NY, USA. Differences between treatments were assessed with a Student's $t$-test, and the $P$-values $<0.05$ were considered statistically significant. Principal component analysis (PCA) was applied to summarize the loadings and 
record information using a reduced number of principal components. Varimax rotation was selected to represent the planar projection of the loadings and scores for the two principal components.

\section{RESULTS}

Growth and extraction yield. In our study, Chlorella stigmatophora displayed the highest growth (Fig. S1 in the Supporting Information) with a specific growth rate $(\mu)$ of $1.30 \cdot \mathrm{d}^{-1}$, while Hemiselmis andersenii showed a growth rate of $0.96 \cdot \mathrm{d}^{-1}$. Biomass yields were 0.48 and $0.32 \mathrm{~g} \mathrm{dw} \cdot \mathrm{L}^{-1}$ for $C$. stigmatophora and $H$. andersenii, respectively. The FTIRATR analysis of microalgal freeze-dried biomass displayed different patterns (Fig. S2 in the Supporting Information) for the species analyzed with major differences in the regions often linked with polysaccharides $\left(900-1,200 \cdot \mathrm{cm}^{-1}\right)$ and lipids $\left(1,745\right.$ and $\left.2,850-3,000 \cdot \mathrm{cm}^{-1}\right)$. Comparing the spectra of raw biomass with the lipophilic extracts before hydrolysis, it was possible to observe specific bands associated with the lipophilic fraction, located at: (i) $3,010 \cdot \mathrm{cm}^{-1}(\nu \mathrm{C}-\mathrm{H}$ of $\mathrm{C}=\mathrm{CH}-$ chains $)$; (ii) 2,954.-2,850 $\mathrm{cm}^{-1}\left(\nu \mathrm{C}-\mathrm{H}\right.$ in $\mathrm{CH}_{3}$ and $\left.\mathrm{CH}_{2} \mathrm{sp}^{3}\right)$; (iii) $1,744 . \mathrm{cm}^{-1}(\nu \mathrm{C}=\mathrm{O}$ in carbonyl groups); (iv) $1,463 . \mathrm{cm}^{-1}\left(\delta \mathrm{CH}_{2}\right)$; and $(\mathrm{v}) 721 \cdot \mathrm{cm}^{-1}\left(\delta \mathrm{CH}_{2}\right.$ rocking; Driver et al. 2015, Forfang et al., 2017). The yields for lipophilic extracts of the marine microalgae strains accounted for $5.76 \pm 0.65 \%$ and $6.50 \pm 0.34 \% \mathrm{dw}$ in C. stigmatophora and H. andersenii, respectively.

Lipophilic characterization. The GC-MS metabolite profile (Fig. 1) of the lipophilic fraction before $(\mathrm{BH})$ and after $(\mathrm{AH})$ alkaline hydrolysis enabled the characterization of up to 71 and $64 \%$ of the total extractable substances from Chlorella stigmatophora and Hemiselmis andersenii. This holistic approach allowed us to identify and quantify a total of 62 metabolites (Table 1). Significant differences $(P<0.05)$ were observed in the metabolites detected for both microalgae before and after alkaline hydrolysis.

Fatty acids. Chlorella stigmatophora and Hemiselmis andersenii fatty acids contained the main set of molecules, with up to 80 and $69 \%$ of the total identified compounds, respectively (Fig. 2). It was possible to observe compositional differences in the fatty acid profiles of both microalgae strains (Fig. 2; Table 1). Hemiselmis andersenii displayed the highest diversity of fatty acids with 25 detected, in comparison to $C$. stigmatophora where only 16 were identified. Monounsaturated fatty acids (MUFA) were the most affected after alkaline hydrolysis, increasing by $54 \%$ and $87 \%$ in $C$. stigmatophora and $H$. andersenii, respectively.

The results in Table 1 show that hexadecanoic

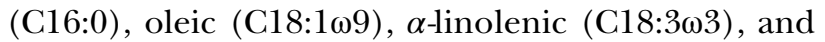

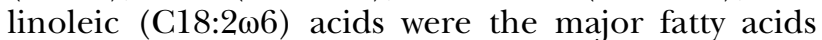
found in Chlorella stigmatophora. In this microalga,

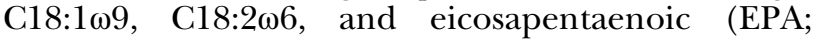
C20:5 $\omega 3$ ) acids increased more than 34\% with alkaline hydrolysis. The four most predominant fatty acids for Hemiselmis andersenii were C16:0, C18:3w3, EPA, and octadecanoic (C18:0) acids. Remarkable

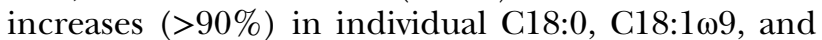
tetradecanoic (C14:0) acid levels were observed for $H$. andersenii lipophilic extracts after hydrolysis.

Chlorella stigmatophora comprised the highest amount of polyunsaturated fatty acids (PUFA)-

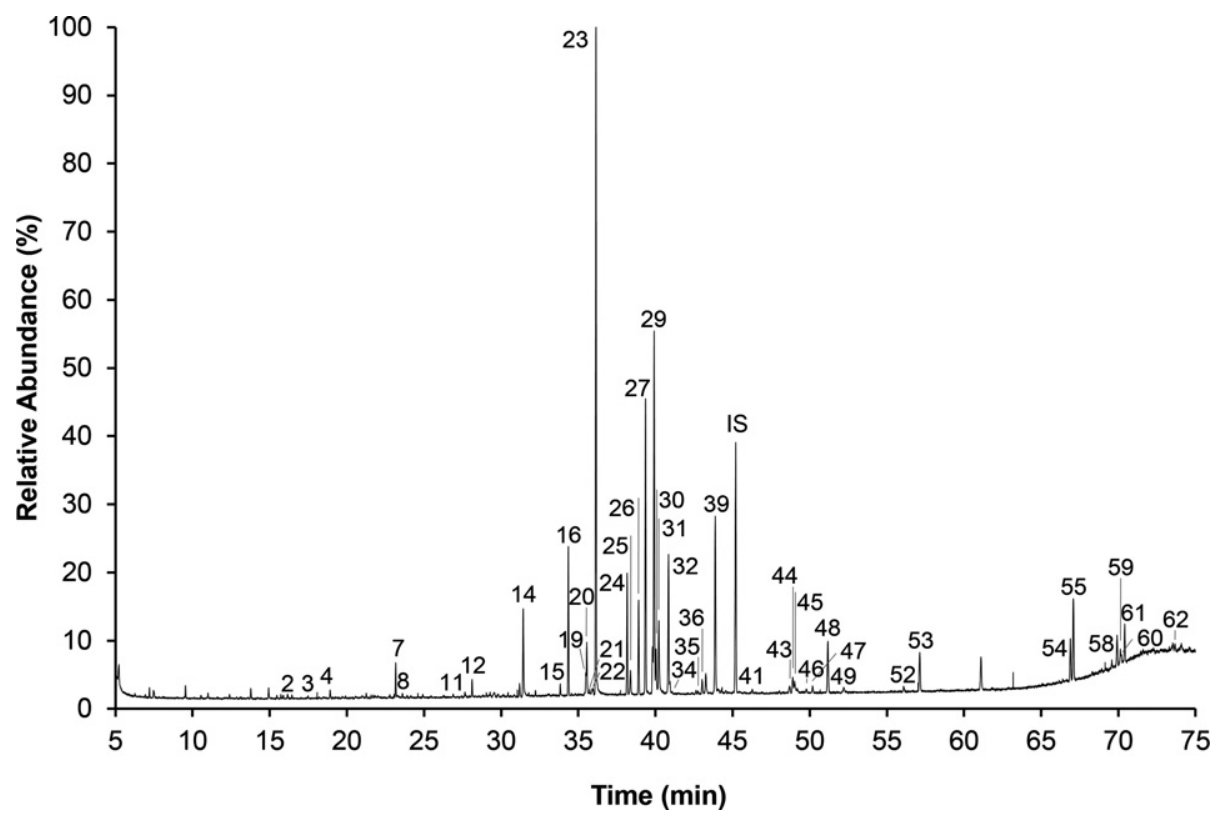

Fig. 1. Chromatogram example of the derivatized Hemiselmis andersenii lipophilic extract before alkaline hydrolysis. The peaks are numbered by their elution order and the correspondent identification is displayed in Table 1. IS, internal standard (tetracosane, $0.20 \mathrm{mg}$ ). 
$10.30 \mathrm{mg} \cdot \mathrm{g} \mathrm{dw}^{-1}$. The main contributors to this

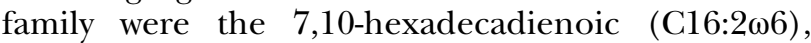

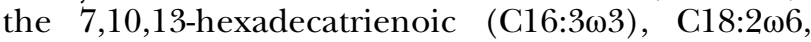

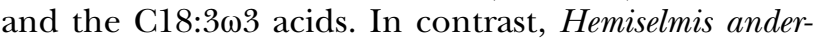
senii displayed the highest level of saturated fatty acids (SFA; $15.18 \mathrm{mg} \cdot \mathrm{g} \mathrm{dw}^{-1}$ ). However, in H. andersenii, it was possible to observe the presence of

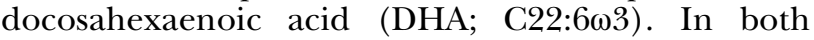
microalgae, the $\Sigma \omega 3 / \Sigma \omega 6$ ratio was higher than one, with $H$. andersenii presenting the highest values when compared to C. stigmatophora (7.26 and 1.24, respectively).

Aliphatic alcohols. In both Chlorella stigmatophora and Hemiselmis andersenii, the aliphatic alcohols were the second most abundant class of lipophilic compounds (Fig. 2), representing up to $24 \%$ and $27 \%$ of the total identified compounds, respectively. In Figure 3, it is possible to observe that the analyzed microalgae had a great diversity of aliphatic alcohols (a total of 17), with the highest diversity verified in $H$. andersenii lipophilic extracts subjected to alkaline hydrolysis being 16 aliphatic alcohols (Fig. 3; Table 1). Between species, it was possible to observe qualitative and quantitative variations. General trends include the predominance of hexadecanol (C16-OH), octadecanol (C18-OH), 9-octadecen-1-ol (C18:1-OH), and phytol (3,7,11,15-tetramethyl-2hexadecen-1-ol), which together accounted for more than $40 \%$ of the total aliphatic alcohols (Fig. 3). Phytol was one of the main aliphatic alcohols affected by the alkaline hydrolysis in C. stigmatophora, yielding a 2.25 times increase after alkaline hydrolysis. This diterpene alcohol alone accounted for up to $15 \%$ and $19 \%$ of the total aliphatic alcohols in C. stigmatophora and $H$. andersenii (Fig. 3), respectively.

Long-chain aliphatic diols $\left(\mathrm{C} 28-(\mathrm{OH})_{2}\right)$ were detected in the composition of the analyzed microalgae. Both octacosane-1,3-diol and octacosane-1,2-diol accounted for up to $12 \%$ and $11 \%$ of total aliphatic alcohols identified in Chlorella stigmatophora and Hemiselmis andersenii (Fig. 3), respectively. The mass spectrum of the octacosane-1,3-diol (Fig. 4a) presented the characteristic ions of an alkane-1,3-diol: $\mathrm{m} / \mathrm{z} \quad 103 \quad\left(\mathrm{TMSOCH}_{2}{ }^{+}\right), \quad 219$ $\left(\left(\mathrm{CH}_{3}\right)_{3} \mathrm{SiOCH}_{2} \mathrm{CH}_{2}\left(\mathrm{CH}_{3}\right)_{3} \mathrm{SiOCH}^{+}\right)$, and $(\mathrm{M}-117$ $\left.\left(\mathrm{TMSOCH}_{2} \mathrm{CH}_{2}\right)\right)^{+}$(Khannoon et al., 2011), while octacosane-1,2-diol (Fig. 4b) presented the diagnostic markers at $\mathrm{m} / \mathrm{z} 73,103,129,147$, and (M-103 $\left.\left(\mathrm{TMSOCH}_{2}\right)\right)^{+}$, which represents the cleavage between carbons 1 and 2 of the alkane-1,2-diol moiety. The sum of long-chain aliphatic alcohols (LCAA; C20 to C36), including the two octacosanediols detected, gives a total of up to $2.21 \mathrm{mg} \cdot \mathrm{g} \mathrm{dw}^{-1}$, in C. stigmatophora, and $3.47 \mathrm{mg} \cdot \mathrm{g} \mathrm{dw}^{-1}$, in H. andersenii.

Sterols and monoglycerides. Both microalgae strains showed a simple sterol profile dominated by $\beta$-sitosterol $(68-70 \%$ of total sterols in Chlorella stigmatophora) and brassicasterol $(58-70 \%$ of total sterols in Hemiselmis andersenii; Table 1). Through Figure $4 \mathrm{~d}$, it is possible to visualize the mass fragmentation of $\beta$-sitosterol. The mass fragmentation corresponds to diagnostic peaks at $\mathrm{m} / \mathrm{z} 129$ and [M$129]^{+}$, which are characteristic of the 3-hydroxy- $\Delta^{5}$ sterol TMS. The observed peaks at $[\mathrm{M}]^{+},[\mathrm{M}-15]^{+}$, and $[\mathrm{M}-90]^{+}$are specific to silylated sterols.

In Hemiselmis andersenii, it was possible to identify seven monoglycerides: five monoacylglycerides and two monoalkylglycerides. In the monoacylglycerides group, two stereoisomers designated as 1- and 2monoacylglycerides were found. The identification of these two isomers was possible by the analysis of the diagnostic markers at $\mathrm{m} / \mathrm{z} 218$ and $[\mathrm{M}-103]^{+}$. Therefore, in 1-monoglycerides, the intensity of the peak at $\mathrm{m} / \mathrm{z} 218$ was lower than that observed in 2monoglycerides, and a high-intensity peak at [M$103]^{+}$was observed, in contrast to 2-monoglycerides. In Figure 4c, it is possible to visualize the mass spectra obtained for 1-monohexadecanoin. The monoglyceride content showed an $82 \%$ reduction yield after alkaline hydrolysis, where only 1-hexadecylglycerol and 1-octadecylglycerol were detected.

Principal component analysis. Principal component analysis (PCA) was performed on the metabolites found in lipophilic extracts of Hemiselmis andersenii and Chlorella stigmatophora to summarize the metabolites species information before and after alkaline hydrolysis. Figure 5 a represents the distribution of the loadings for the species studied before and after hydrolysis in a two components model. The first component (PC1) accounted for $50 \%$ of variance, whereas the second (PC2) accounted for $40 \%$, which together explained $90 \%$ of the total variance. The variables strongly associated with positive values of factor 1 were: tetradecanoic acid (14), heptadecanoic acid (25), eicosanoic acid (41), vaccenic acid (31), 10-nonadecenoic acid (36), eicosapentaenoic acid (39), aliphatic alcohols (AA), octacosane-1,3-diol (59), 2,6-bis (1,1-dimethylethyl)phenol (7), and dehydroabietic acid (38). While 10-hydroxyoctadecanoic acid (43), docosanoic acid (49), methyl-4,7,10,13-hexadecatetraenoate (22), 7,10,13,16,19-docosapentaenoic acid (45), eicosanol (40), monoglycerides (MG), 1-Monohexadecanoin (48), 2-Monohexadecanoin (47), 1monooctadecanoin (53), 2-monooctadecanoin (52), 2-methyltetradecane (4), and methylsuccinic acid (12) were positively correlated to factor 2.

The score plot (Fig. 5b) provides evidence for clear chemical divergence in the GC-MS-based metabolite profile of Hemiselmis andersenii before $(\mathrm{BH})$ and after $(\mathrm{AH})$ hydrolysis. PCA results showed that Chlorella stigmatophora samples were richer in PUFA, oleic acid, $\beta$-sitosterol, and LA. While $H$. andersenii $\mathrm{BH}$ presented higher levels of sterols, monoglycerides, and DHA, the $H$. andersenii $\mathrm{AH}$ had higher amounts of the total identified compounds and SFA.

PC1 separated the Chlorella stigmatophora samples from Hemiselmis andersenii, indicating that the 
TABLE 1. Lipophilic profile ( $\mathrm{mg} \cdot \mathrm{g}$ microalgal $\left.\mathrm{dw}^{-1}\right)$ of Chlorella stigmatophora and Hemiselmis andersenii before (BH) and after (AH) hydrolysis

\begin{tabular}{|c|c|c|c|c|c|c|c|}
\hline \multirow[b]{2}{*}{ No } & \multirow[b]{2}{*}{ Identified Compounds } & \multirow[b]{2}{*}{ Formula } & \multirow[b]{2}{*}{ MW } & \multicolumn{2}{|c|}{ Chlorella stigmatophora } & \multicolumn{2}{|c|}{ Hemiselmis andersenii } \\
\hline & & & & $\mathrm{BH}$ & $\mathrm{AH}$ & BH & $\mathrm{AH}$ \\
\hline \multirow[t]{2}{*}{ Fatty acids } & & & & $22.22 \pm 0.41^{\mathrm{a}}$ & $28.62 \pm 0.23^{b}$ & $18.30 \pm 0.22^{\mathrm{a}}$ & $28.10 \pm 2.67^{b}$ \\
\hline & Saturated & & & $7.34 \pm 0.04^{\mathrm{a}}$ & $8.34 \pm 0.05^{b}$ & $8.17 \pm 0.12^{\mathrm{a}}$ & $15.18 \pm 1.69^{b}$ \\
\hline 3 & Nonanoic acid & $\mathrm{C}_{9} \mathrm{H}_{18} \mathrm{O}_{2}$ & 230 & $0.52 \pm 0.00^{\mathrm{a}}$ & $0.52 \pm 0.00^{\mathrm{a}}$ & $0.51 \pm 0.01^{\mathrm{a}}$ & $0.76 \pm 0.02^{\mathrm{b}}$ \\
\hline 5 & Decanoic acid & $\mathrm{C}_{10} \mathrm{H}_{20} \mathrm{O}_{2}$ & 244 & n.d. & n.d. & n.d. & $0.75 \pm 0.02$ \\
\hline 10 & Dodecanoic acid & $\mathrm{C}_{12} \mathrm{H}_{24} \mathrm{O}_{2}$ & 272 & n.d. & $0.54 \pm 0.00$ & n.d. & $0.76 \pm 0.03$ \\
\hline 14 & Tetradecanoic acid & $\mathrm{C}_{14} \mathrm{H}_{28} \mathrm{O}_{2}$ & 300 & $0.57 \pm 0.00^{\mathrm{a}}$ & $0.60 \pm 0.00^{\mathrm{b}}$ & $0.72 \pm 0.12^{\mathrm{a}}$ & $1.39 \pm 0.06^{\mathrm{b}}$ \\
\hline 15 & Pentadecanoic acid & $\mathrm{C}_{15} \mathrm{H}_{30} \mathrm{O}_{2}$ & 314 & $0.53 \pm 0.00^{\mathrm{a}}$ & $0.53 \pm 0.00^{\mathrm{a}}$ & $0.54 \pm 0.01^{\mathrm{a}}$ & $0.81 \pm 0.02^{\mathrm{b}}$ \\
\hline 23 & Hexadecanoic acid & $\mathrm{C}_{16} \mathrm{H}_{32} \mathrm{O}_{2}$ & 328 & $3.53 \pm 0.03^{\mathrm{a}}$ & $4.50 \pm 0.04^{\mathrm{b}}$ & $3.00 \pm 0.06^{\mathrm{a}}$ & $5.51 \pm 0.86^{\mathrm{b}}$ \\
\hline 25 & Heptadecanoic acid & $\mathrm{C}_{17} \mathrm{H}_{34} \mathrm{O}_{2}$ & 342 & $0.61 \pm 0.01^{\mathrm{a}}$ & $0.56 \pm 0.00^{\mathrm{b}}$ & $0.65 \pm 0.04^{\mathrm{a}}$ & $0.89 \pm 0.01^{\mathrm{b}}$ \\
\hline 32 & Octadecanoic acid & $\mathrm{C}_{18} \mathrm{H}_{36} \mathrm{O}_{2}$ & 356 & $1.07 \pm 0.02^{\mathrm{a}}$ & $1.09 \pm 0.01^{\mathrm{a}}$ & $1.15 \pm 0.06^{\mathrm{a}}$ & $2.77 \pm 0.88^{\mathrm{b}}$ \\
\hline 43 & $\begin{array}{l}\text { 10-Hydroxyoctadecanoic } \\
\text { acid }\end{array}$ & $\mathrm{C}_{18} \mathrm{H}_{36} \mathrm{O}_{3}$ & 444 & n.d. & n.d. & $0.55 \pm 0.01^{\mathrm{a}}$ & n.d. \\
\hline 37 & Nonadecanoic acid & $\mathrm{C}_{19} \mathrm{H}_{38} \mathrm{O}_{2}$ & 370 & n.d. & n.d. & n.d. & $0.77 \pm 0.02$ \\
\hline 41 & Eicosanoic acid & $\mathrm{C}_{20} \mathrm{H}_{40} \mathrm{O}_{2}$ & 384 & n.d. & n.d. & $0.52 \pm 0.00^{\mathrm{a}}$ & $0.77 \pm 0.01^{\mathrm{b}}$ \\
\hline \multirow[t]{2}{*}{49} & Docosanoic acid & $\mathrm{C}_{22} \mathrm{H}_{44} \mathrm{O}_{2}$ & 412 & n.d. & n.d. & $0.55 \pm 0.01$ & n.d. \\
\hline & Monounsaturated & & & $6.46 \pm 0.14^{\mathrm{a}}$ & $9.98 \pm 0.14^{\mathrm{b}}$ & $3.77 \pm 0.12^{\mathrm{a}}$ & $7.06 \pm 0.12^{\mathrm{b}}$ \\
\hline 20 & 9-Hexadecenoic acid $^{1}$ & $\mathrm{C}_{16} \mathrm{H}_{30} \mathrm{O}_{2}$ & 326 & $1.43 \pm 0.00^{\mathrm{a}}$ & $1.62 \pm 0.01^{\mathrm{b}}$ & $1.13 \pm 0.09^{\mathrm{a}}$ & $1.59 \pm 0.04^{\mathrm{b}}$ \\
\hline 21 & 7-Hexadecenoic acid & $\mathrm{C}_{16} \mathrm{H}_{30} \mathrm{O}_{2}$ & 326 & $0.70 \pm 0.01^{\mathrm{a}}$ & $0.79 \pm 0.01^{\mathrm{b}}$ & $0.51 \pm 0.00^{\mathrm{a}}$ & $0.83 \pm 0.01^{\mathrm{b}}$ \\
\hline 31 & Vaccenic acid & $\mathrm{C}_{18} \mathrm{H}_{34} \mathrm{O}_{2}$ & 354 & $0.65 \pm 0.01^{\mathrm{a}}$ & $0.63 \pm 0.00^{\mathrm{b}}$ & $0.87 \pm 0.04^{\mathrm{a}}$ & $1.51 \pm 0.17^{\mathrm{b}}$ \\
\hline 30 & Oleic acid & $\mathrm{C}_{18} \mathrm{H}_{34} \mathrm{O}_{2}$ & 354 & $3.68 \pm 0.16^{\mathrm{a}}$ & $6.94 \pm 0.03^{\mathrm{b}}$ & $0.65 \pm 0.04^{\mathrm{a}}$ & $1.29 \pm 0.04^{\mathrm{b}}$ \\
\hline 33 & 7-Nonadecenoic acid & $\mathrm{C}_{19} \mathrm{H}_{36} \mathrm{O}_{2}$ & 368 & n.d. & n.d. & n.d. & $0.82 \pm 0.03$ \\
\hline \multirow[t]{2}{*}{36} & 10-Nonadecenoic acid & $\mathrm{C}_{19} \mathrm{H}_{36} \mathrm{O}_{2}$ & 368 & n.d. & n.d. n.d. & $0.60 \pm 0.03^{\mathrm{a}}$ & $1.02 \pm 0.03^{\mathrm{b}}$ \\
\hline & Polyunsaturated & & & $8.41 \pm 0.24^{\mathrm{a}}$ & $10.30 \pm 0.19^{\mathrm{b}}$ & $6.35 \pm 0.11^{\mathrm{a}}$ & $7.67 \pm 0.10^{\mathrm{b}}$ \\
\hline 19 & 7,12-Hexadienoic acid & $\mathrm{C}_{16} \mathrm{H}_{28} \mathrm{O}_{2}$ & 324 & n.d. & n.d. & $0.57 \pm 0.01^{\mathrm{a}}$ & $0.80 \pm 0.01^{\mathrm{b}}$ \\
\hline 17 & $\begin{array}{l}\text { 7,10-Hexadecadienoic } \\
\text { acid }\end{array}$ & $\mathrm{C}_{16} \mathrm{H}_{28} \mathrm{O}_{2}$ & 324 & $1.64 \pm 0.03^{\mathrm{a}}$ & $1.70 \pm 0.05^{\mathrm{a}}$ & n.d. & n.d. \\
\hline 18 & $\begin{array}{l}7,10,13- \\
\text { Hexadecatrienoic acid }\end{array}$ & $\mathrm{C}_{16} \mathrm{H}_{26} \mathrm{O}_{2}$ & 322 & $1.94 \pm 0.02^{\mathrm{a}}$ & $2.01 \pm 0.02^{\mathrm{b}}$ & n.d. & n.d. \\
\hline 22 & $\begin{array}{l}\text { Methyl-4,7,10,13- } \\
\text { hexadecatetraenoate }\end{array}$ & $\mathrm{C}_{17} \mathrm{H}_{26} \mathrm{O}_{2}$ & 262 & n.d. & n.d. & $0.52 \pm 0.00$ & n.d. \\
\hline 28 & 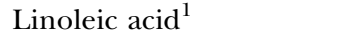 & $\mathrm{C}_{18} \mathrm{H}_{32} \mathrm{O}_{2}$ & 352 & $2.11 \pm 0.13^{\mathrm{a}}$ & $3.21 \pm 0.15^{\mathrm{b}}$ & $0.70 \pm 0.02^{\mathrm{a}}$ & $1.22 \pm 0.03^{\mathrm{b}}$ \\
\hline 29 & $\alpha$-Linolenic acid & $\mathrm{C}_{18} \mathrm{H}_{30} \mathrm{O}_{2}$ & 350 & $2.19 \pm 0.14^{\mathrm{a}}$ & $2.67 \pm 0.02^{\mathrm{b}}$ & $2.05 \pm 0.05^{\mathrm{a}}$ & $2.55 \pm 0.23^{\mathrm{b}}$ \\
\hline 39 & Eicosapentaenoic acid & $\mathrm{C}_{20} \mathrm{H}_{30} \mathrm{O}_{2}$ & 374 & $0.53 \pm 0.00^{\mathrm{a}}$ & $0.71 \pm 0.07^{\mathrm{b}}$ & $1.35 \pm 0.04^{\mathrm{a}}$ & $1.86 \pm 0.17^{\mathrm{b}}$ \\
\hline 45 & $\begin{array}{l}7,10,13,16,19- \\
\text { Docosapentaenoic acid }\end{array}$ & $\mathrm{C}_{22} \mathrm{H}_{34} \mathrm{O}_{2}$ & 402 & n.d. & n.d. & $0.57 \pm 0.01$ & n.d. \\
\hline 44 & Docosahexaenoic acid & $\mathrm{C}_{22} \mathrm{H}_{32} \mathrm{O}_{2}$ & 400 & n.d. & n.d. & $0.60 \pm 0.01^{\mathrm{a}}$ & $0.91 \pm 0.04$ \\
\hline Aliphatic Alcohols & & & & $7.15 \pm 0.05^{\mathrm{a}}$ & $6.81 \pm 0.02^{\mathrm{b}}$ & $8.19 \pm 0.47^{\mathrm{a}}$ & $10.95 \pm 0.23^{\mathrm{b}}$ \\
\hline 1 & Octanol & $\mathrm{C}_{8} \mathrm{H}_{18} \mathrm{O}$ & 202 & n.d. & n.d. & n.d. & $0.61 \pm 0.02$ \\
\hline 6 & Undecanol & $\mathrm{C}_{11} \mathrm{H}_{24} \mathrm{O}$ & 244 & $0.43 \pm 0.00^{\mathrm{a}}$ & $0.42 \pm 0.00^{\mathrm{b}}$ & n.d. & $0.61 \pm 0.02$ \\
\hline 9 & Dodecanol & $\mathrm{C}_{12} \mathrm{H}_{26} \mathrm{O}$ & 258 & n.d. & n.d. & n.d. & $0.61 \pm 0.02$ \\
\hline 11 & Tridecanol & $\mathrm{C}_{13} \mathrm{H}_{28} \mathrm{O}$ & 272 & $0.42 \pm 0.00^{\mathrm{a}}$ & $0.42 \pm 0.00^{\mathrm{a}}$ & $0.42 \pm 0.01^{\mathrm{a}}$ & $0.64 \pm 0.02^{\mathrm{b}}$ \\
\hline 13 & Tetradecanol & $\mathrm{C}_{14} \mathrm{H}_{30} \mathrm{O}$ & 286 & $0.47 \pm 0.00^{\mathrm{a}}$ & $0.42 \pm 0.00^{\mathrm{b}}$ & n.d. & $0.62 \pm 0.03$ \\
\hline 16 & Hexadecanol & $\mathrm{C}_{16} \mathrm{H}_{34} \mathrm{O}$ & 314 & $1.04 \pm 0.01^{\mathrm{a}}$ & $0.71 \pm 0.01^{\mathrm{b}}$ & $0.99 \pm 0.12^{\mathrm{a}}$ & $0.98 \pm 0.04^{\mathrm{a}}$ \\
\hline 26 & Octadecanol & $\mathrm{C}_{18} \mathrm{H}_{38} \mathrm{O}$ & 342 & $1.48 \pm 0.01^{\mathrm{a}}$ & $0.66 \pm 0.00^{\mathrm{b}}$ & $0.79 \pm 0.06^{\mathrm{a}}$ & $0.87 \pm 0.01^{\mathrm{a}}$ \\
\hline 24 & 9-Octadecen-1-ol & $\mathrm{C}_{18} \mathrm{H}_{36} \mathrm{O}$ & 340 & $0.81 \pm 0.01^{\mathrm{a}}$ & $0.95 \pm 0.01^{\mathrm{b}}$ & $0.95 \pm 0.17^{\mathrm{a}}$ & $1.00 \pm 0.05^{\mathrm{a}}$ \\
\hline 40 & Eicosanol & $\mathrm{C}_{20} \mathrm{H}_{40} \mathrm{O}$ & 368 & $0.58 \pm 0.01^{\mathrm{a}}$ & $0.42 \pm 0.00^{\mathrm{b}}$ & n.d. & n.d. \\
\hline 34 & 11-Eicosen-1-ol & $\mathrm{C}_{20} \mathrm{H}_{38} \mathrm{O}$ & 366 & n.d. & n.d. & $0.44 \pm 0.01$ & n.d. \\
\hline 46 & Docosanol & $\mathrm{C}_{22} \mathrm{H}_{44} \mathrm{O}$ & 396 & n.d. & n.d. & $0.42 \pm 0.00^{\mathrm{a}}$ & $0.62 \pm 0.02^{\mathrm{b}}$ \\
\hline 54 & Octacosanol & $\mathrm{C}_{28} \mathrm{H}_{58} \mathrm{O}$ & 482 & $0.45 \pm 0.01^{\mathrm{a}}$ & $0.49 \pm 0.01^{\mathrm{b}}$ & $0.62 \pm 0.04^{\mathrm{a}}$ & $0.67 \pm 0.04^{\mathrm{a}}$ \\
\hline 61 & Triacontanol & $\mathrm{C}_{30} \mathrm{H}_{62} \mathrm{O}$ & 510 & $0.54 \pm 0.01^{\mathrm{a}}$ & $0.45 \pm 0.00^{\mathrm{b}}$ & $0.61 \pm 0.06^{\mathrm{a}}$ & $0.70 \pm 0.02^{\mathrm{b}}$ \\
\hline 62 & Dotriacontanol & $\mathrm{C}_{32} \mathrm{H}_{66} \mathrm{O}$ & 538 & n.d. & n.d. & $0.45 \pm 0.01^{\mathrm{a}}$ & $0.62 \pm 0.02^{\mathrm{b}}$ \\
\hline 59 & Octacosane-1,3-diol & $\mathrm{C}_{28} \mathrm{H}_{58} \mathrm{O}_{2}$ & 570 & n.d. & $0.42 \pm 0.00^{\mathrm{b}}$ & $0.49 \pm 0.03^{\mathrm{a}}$ & $0.66 \pm 0.02^{\mathrm{b}}$ \\
\hline 60 & Octacosane-1,2-diol & $\mathrm{C}_{28} \mathrm{H}_{58} \mathrm{O}_{2}$ & 570 & $0.45 \pm 0.01^{\mathrm{a}}$ & $0.43 \pm 0.00^{\mathrm{b}}$ & $0.44 \pm 0.01$ & n.d. \\
\hline 27 & Phytol & $\mathrm{C}_{20} \mathrm{H}_{40} \mathrm{O}$ & 368 & $0.48 \pm 0.00^{\mathrm{a}}$ & $1.02 \pm 0.01^{\mathrm{b}}$ & $1.57 \pm 0.04^{\mathrm{a}}$ & $1.74 \pm 0.18^{\mathrm{a}}$ \\
\hline Sterols & & & & $0.73 \pm 0.01^{\mathrm{a}}$ & $0.62 \pm 0.01^{\mathrm{b}}$ & $1.09 \pm 0.06^{\mathrm{a}}$ & $0.77 \pm 0.07^{\mathrm{b}}$ \\
\hline 55 & Brassicasterol & $\mathrm{C}_{28} \mathrm{H}_{46} \mathrm{O}$ & 470 & n.d. & n.d. & $0.76 \pm 0.06^{\mathrm{a}}$ & $0.45 \pm 0.04^{\mathrm{b}}$ \\
\hline 56 & 24-Methylenecholesterol & $\mathrm{C}_{28} \mathrm{H}_{46} \mathrm{O}$ & 472 & $0.10 \pm 0.01^{\mathrm{a}}$ & $0.09 \pm 0.00^{\mathrm{a}}$ & n.d. & n.d. \\
\hline 57 & Campesterol & $\mathrm{C}_{28} \mathrm{H}_{48} \mathrm{O}$ & 472 & $0.12 \pm 0.01^{\mathrm{a}}$ & $0.10 \pm 0.00^{\mathrm{b}}$ & n.d. & n.d. \\
\hline 58 & $\beta$-Sitosterol & $\mathrm{C}_{29} \mathrm{H}_{50} \mathrm{O}$ & 486 & $0.51 \pm 0.01^{\mathrm{a}}$ & $0.42 \pm 0.01^{\mathrm{b}}$ & $0.33 \pm 0.05^{\mathrm{a}}$ & $0.32 \pm 0.04^{\mathrm{a}}$ \\
\hline Monoglycerides & & & & n.d. & n.d. & $1.11 \pm 0.07^{\mathrm{a}}$ & $0.20 \pm 0.03^{\mathrm{b}}$ \\
\hline 35 & 1-Monotridecanoin & $\mathrm{C}_{16} \mathrm{H}_{32} \mathrm{O}_{4}$ & 432 & n.d. & n.d. & $0.04 \pm 0.01$ & n.d. \\
\hline 48 & 1-Monohexadecanoin & $\mathrm{C}_{19} \mathrm{H}_{38} \mathrm{O}_{4}$ & 474 & n.d. & n.d. & $0.49 \pm 0.01$ & n.d. \\
\hline 47 & 2-Monohexadecanoin & $\mathrm{C}_{19} \mathrm{H}_{38} \mathrm{O}_{4}$ & 474 & n.d. & n.d. & $0.06 \pm 0.01$ & n.d. \\
\hline
\end{tabular}


TABLE 1. (continued)

\begin{tabular}{|c|c|c|c|c|c|c|c|}
\hline \multirow[b]{2}{*}{ No } & \multirow[b]{2}{*}{ Identified Compounds } & \multirow[b]{2}{*}{ Formula } & \multirow[b]{2}{*}{ MW } & \multicolumn{2}{|c|}{ Chlorella stigmatophora } & \multicolumn{2}{|c|}{ Hemiselmis andersenii } \\
\hline & & & & BH & $\mathrm{AH}$ & $\mathrm{BH}$ & $\mathrm{AH}$ \\
\hline 53 & 1-Monooctadecanoin & $\mathrm{C}_{21} \mathrm{H}_{42} \mathrm{O}_{4}$ & 502 & n.d. & n.d. & $0.47 \pm 0.07$ & n.d. \\
\hline 52 & 2-Monooctadecanoin & $\mathrm{C}_{21} \mathrm{H}_{42} \mathrm{O}_{4}$ & 502 & n.d. & n.d. & $0.05 \pm 0.01$ & n.d. \\
\hline 42 & 1-Hexadecylglycerol & $\mathrm{C}_{19} \mathrm{H}_{40} \mathrm{O}_{3}$ & 460 & n.d. & n.d. & n.d. & $0.10 \pm 0.02$ \\
\hline 51 & 1-Octadecylglycerol & $\mathrm{C}_{21} \mathrm{H}_{44} \mathrm{O}_{3}$ & 488 & n.d. & n.d. & n.d. & $0.10 \pm 0.01$ \\
\hline Others & & & & $0.08 \pm 0.01^{\mathrm{a}}$ & $0.02 \pm 0.00^{\mathrm{b}}$ & $1.89 \pm 0.03^{\mathrm{a}}$ & $0.85 \pm 0.07^{\mathrm{b}}$ \\
\hline 2 & $\begin{array}{l}\text { 2,4,6,8-Tetramethyl-1- } \\
\text { undecene }\end{array}$ & $\mathrm{C}_{15} \mathrm{H}_{30}$ & 210 & n.d. & n.d. & $0.11 \pm 0.00$ & n.d. \\
\hline 4 & 2-Methyltetradecane & $\mathrm{C}_{15} \mathrm{H}_{32}$ & 212 & n.d. & n.d. & $0.06 \pm 0.01$ & n.d. \\
\hline 8 & 3-Methyl-4-nonadecene & $\mathrm{C}_{20} \mathrm{H}_{40}$ & 280 & n.d. & n.d. & $0.04 \pm 0.01^{\mathrm{a}}$ & $0.43 \pm 0.06^{\mathrm{b}}$ \\
\hline 7 & $\begin{array}{l}\text { 2,6-bis (1,1- } \\
\text { Dimethylethyl)phenol }\end{array}$ & $\mathrm{C}_{14} \mathrm{H}_{22} \mathrm{O}$ & 278 & $0.08 \pm 0.01^{\mathrm{a}}$ & $0.02 \pm 0.00^{\mathrm{b}}$ & $0.22 \pm 0.03^{\mathrm{a}}$ & $0.04 \pm 0.01^{\mathrm{b}}$ \\
\hline 12 & Methylsuccinic acid & $\mathrm{C}_{5} \mathrm{H}_{8} \mathrm{O}_{4}$ & 276 & n.d. & n.d. & $0.09 \pm 0.02^{\mathrm{a}}$ & $0.16 \pm 0.00^{\mathrm{b}}$ \\
\hline 38 & Dehydroabietic acid & $\mathrm{C}_{20} \mathrm{H}_{28} \mathrm{O}_{2}$ & 372 & n.d. & n.d. & $1.37 \pm 0.06^{\mathrm{a}}$ & $0.11 \pm 0.01^{\mathrm{b}}$ \\
\hline 50 & Pinoresinol & $\mathrm{C}_{20} \mathrm{H}_{22} \mathrm{O}_{6}$ & 502 & n.d. & n.d. & n.d. & $0.16 \pm 0.01$ \\
\hline$\Sigma \omega 3 / \Sigma \omega 6$ & & & & $1.24 \pm 0.02^{\mathrm{a}}$ & $1.10 \pm 0.03^{\mathrm{b}}$ & $7.26 \pm 0.14^{\mathrm{a}}$ & $4.65 \pm 0.14^{\mathrm{b}}$ \\
\hline $\begin{array}{l}\text { Total identified } \\
\left(\mathrm{mg}^{-1} \mathrm{~g} \text { microalgal }\right. \\
\left.\mathrm{dw}^{-1}\right)\end{array}$ & & & & $30.18 \pm 0.39^{\mathrm{a}}$ & $36.07 \pm 0.39^{\mathrm{b}}$ & $30.59 \pm 0.49^{\mathrm{a}}$ & $41.89 \pm 2.68^{\mathrm{b}}$ \\
\hline
\end{tabular}

Values (means \pm SD of four replicates) in the same row, not sharing a common superscript are significantly different $(P<0.05)$. Compounds are numbered by their elution order (Fig. 1). All the compounds containing hydroxyl and/or carboxyl groups are identified as the correspondent trimethylsilyl derivatives. MW, Molecular weight of compounds after silylation. ${ }^{1}$ Contains cis and trans isomers. n.d., non detected. $d w$, dry weight. In bold are the main lipophilic classes.

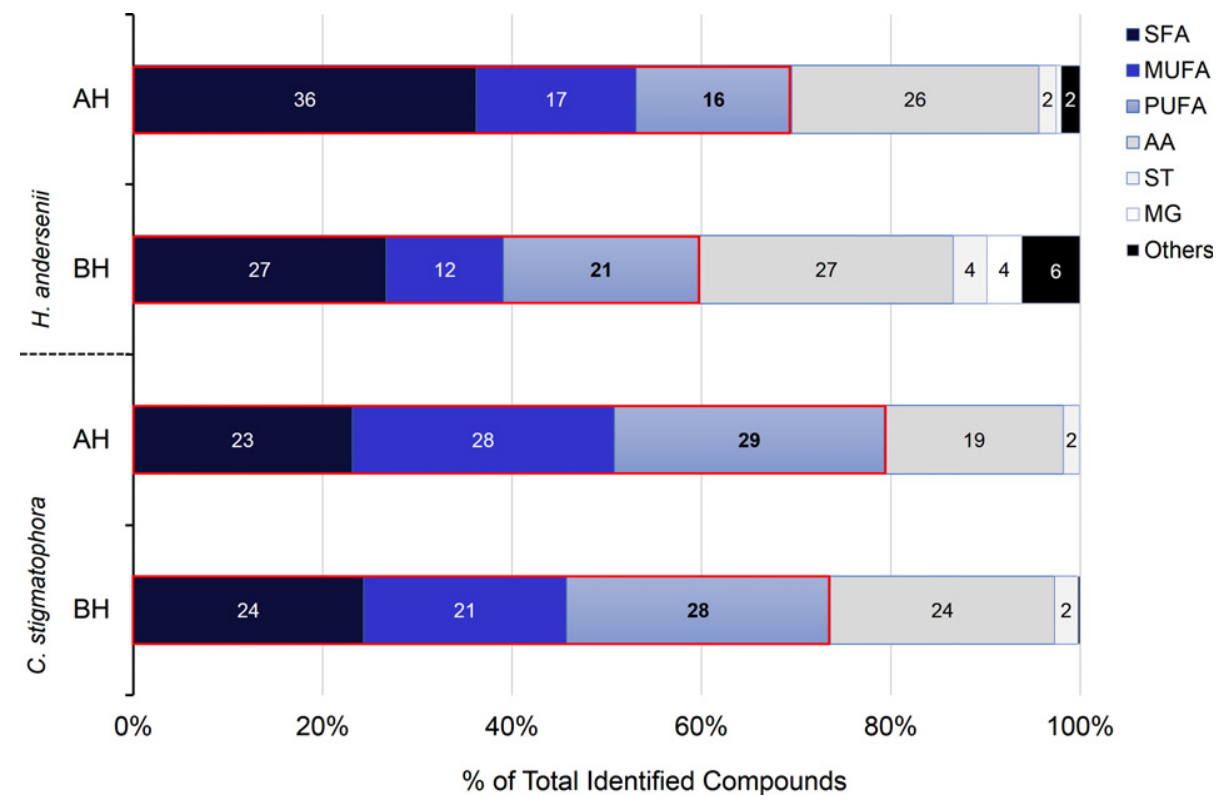

Fig. 2. Relative abundance of the main classes of compounds identified in the lipophilic extracts of the two microalgae studied before $(\mathrm{BH})$ and after $(\mathrm{AH})$ alkaline hydrolysis. SFA, saturated fatty acids; MUFA, monounsaturated fatty acids; PUFA, polyunsaturated fatty acids; AA, aliphatic alcohols; ST, sterols; MG, monoglycerides. Highlighted boxes are the percentage of total fatty acids. [Colour figure can be viewed at wileyonlinelibrary.com]

lipophilic profile of both microalgae had unique features that could be further investigated as chemotaxonomic markers. Namely, the compounds 7,12-hexadienoic acid (19), 7,10,13-hexadecatrienoic acid (18), eicosanol (40), 24-methylenecholesterol (56) and campesterol (57) that were only present in C. stigmatophora samples and the 10-nonadecenoic acid (36), 7,12-hexadienoic acid (19), DHA (44), docosanol (46), and brassicasterol (55) that were only found in $H$. andersenii samples.

\section{DISCUSSION}

Growth and extraction yield. To explore the potential of microalgae as sustainable sources of valueadded compounds, it is essential to assess 


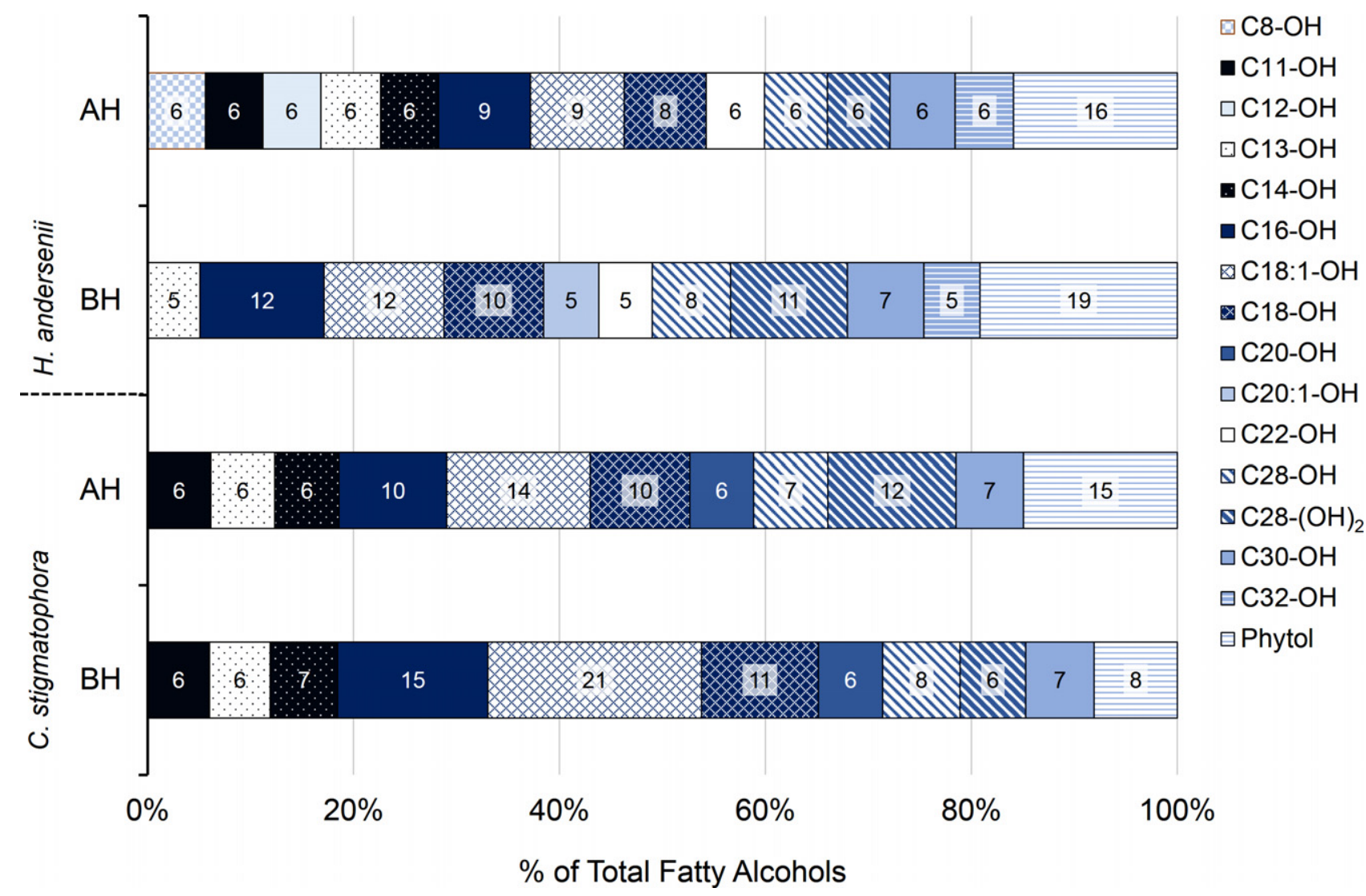

FIG. 3. Aliphatic alcohols proportion in Chlorella stigmatophora and Hemiselmis andersenii before (BH) and after (AH) alkaline hydrolysis. [Colour figure can be viewed at wileyonlinelibrary.com]

microalgae growth characteristics, biomass yield, and desired product content. The highest specific growth rate verified for Chlorella stigmatophora is in accordance with previous data that showed Chlorella as fast-growing strains (Peltomaa et al., 2017). In contrast to Chlorella, Cryptophytes are known to grow slowly $\left(\mu<0.80 \cdot \mathrm{d}^{-1}\right)$, but in suitable conditions, some strains can achieve higher growth rates (Peltomaa et al. 2017).

The nature of microalgae cell covering is known to affect their shear resistance and cellular biomass weight (Borowitzka 2013). As a result, the absence of a heavy and rigid cell wall structure in Hemiselmis andersenii makes cells lighter and easier to break and process for further commercial purposes than Chlorella stigmatophora (Peltomaa et al. 2017). This observation explains the fact that the biomass yield verified for $H$. andersenii was low, despite its growth rate (estimated from cellular concentration) being high. Biomass values obtained for C. stigmatophora were $45 \%$ higher than those previously reported by Slocombe et al. (2015) that revealed values near to $0.33 \mathrm{~g} \mathrm{dw} \cdot \mathrm{L}^{-1}$.

The amount of lipophilic substances in Chlorella stigmatophora was lower than that previously obtained by Slocombe et al. (2015). The low lipophilic extracts' yields might be explained by Chlorella strains' growth conditions and the cell physiological status. Although there is no information regarding Hemiselmis species lipophilic contents, the values observed in the present study were lower than those determined by Huerlimann et al. (2010) for another Cryptophyta (Rhodomonas sp.) cultivated in $\mathrm{f} / 2$ growth medium, $13 \% \mathrm{dw}$.

Lipophilic characterization. In the present study, microalgae were scaled up in the same conditions as the inoculum and were collected at the late exponential phase, instead of the stationary phase. The low yields of lipophilic extracts-which in turn influence fatty acids, aliphatic alcohols, sterols, and other compound contents-might be explained by the assumption that lipid accumulation is often triggered by environmental fluctuations in microalgae growth systems (e.g., nutrient deficiency, light saturation, and growth stage; Sukenic 1991). Thus, the growth conditions employed were not ideal for eliciting marine microalgae carbon partitioning toward lipid accumulation.

Fatty acids. Neutral lipids like triacylglycerols are mainly constructed by SFA and MUFA (Guiheneuf and Stengel 2013). Therefore, the significant MUFA increase after alkaline hydrolysis might indicate that acylglycerols are mostly constituted by these fatty

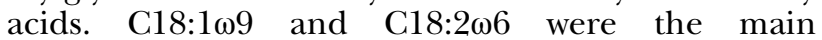


a)

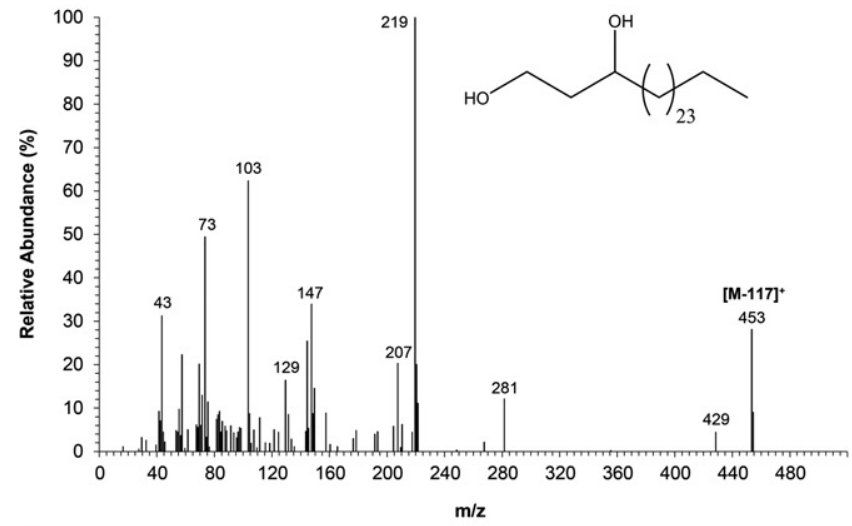

b)

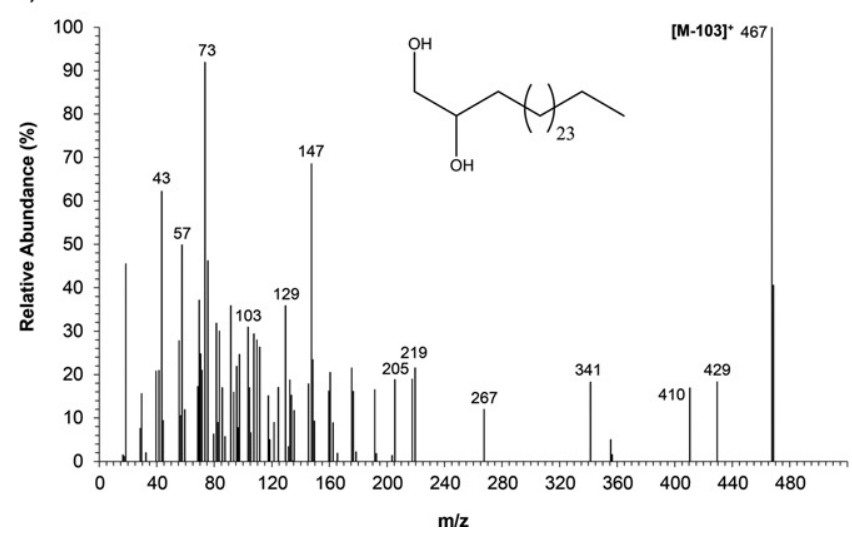

c)

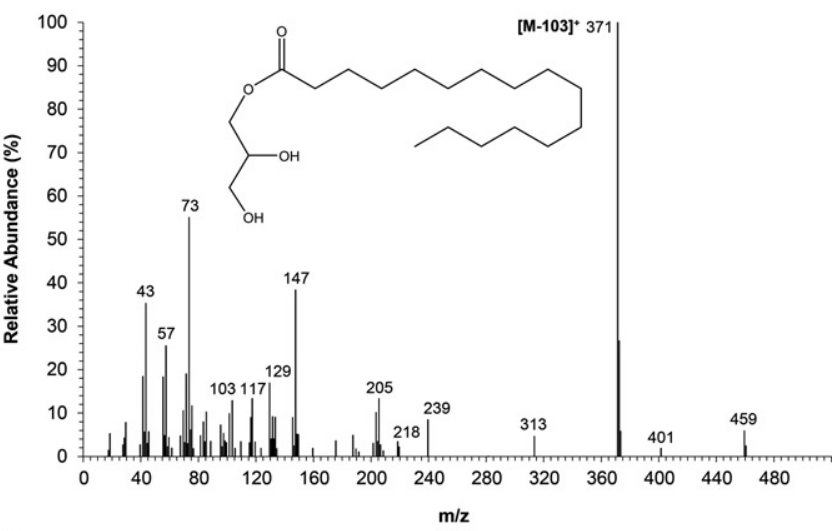

d)

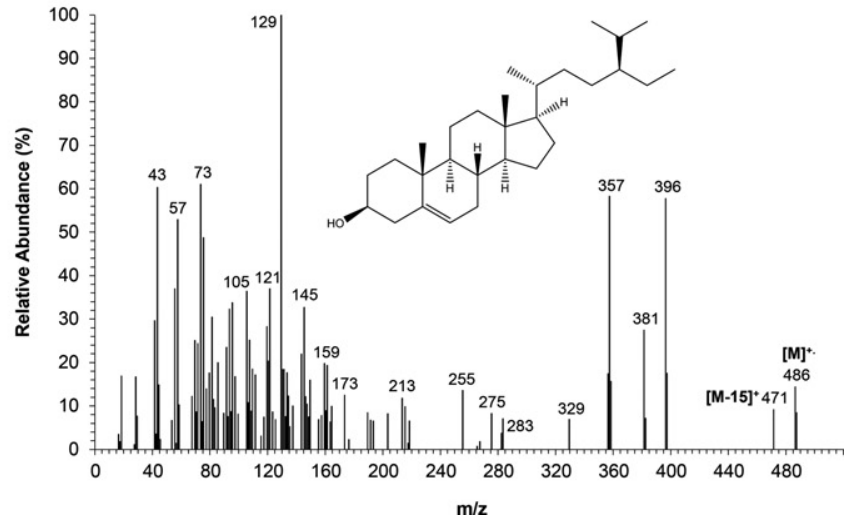

FIG. 4. Mass spectra for four of the identified compounds: a) octacosane-1,3-diol (peak 59); b) octacosane-1,2-diol (peak 60); c) 1Monohexadecanoin (peak 48); and d) $\beta$-Sitosterol (peak 58), as trimethylsilyl derivatives.

components of the esterified fraction of Chlorella

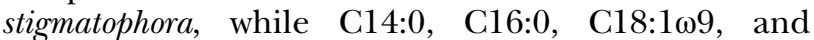
C18:2 $\omega 6$ were the main esterified fatty acids for Hemiselmis andersenii.

In the wide chemical diversity of microalgae, some lipids are ubiquitous, such as C14:0, C16:0, and C18:0 fatty acids, while others are species specific, being useful as biomarkers (Volkman et al. 1998, Taipale et al. 2016). For instance, chlorophytes are recognized to have a predominance of

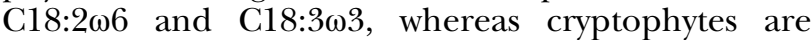
known for containing long-chain PUFA (EPA and

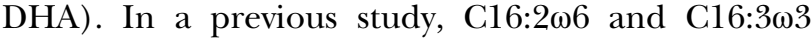
showed to be useful biomarkers for Chlorophytes (Taipale et al. 2016).

PUFA have shown to be effective in preventing or treating several diseases (Lum et al. 2013). In the European Union, a remarkable number of health claims have been authorized based on the essentiality of $\omega 3$ fatty acids (Bucchini 2019). When declaring that a product is a source of $\omega 3$ fatty acids, one of these two requirements has to be fulfilled: (i) must contain $0.3 \mathrm{~g}$ of $\alpha$-linolenic per $100 \mathrm{~g}$ of product or (ii) EPA + DHA must be equal or higher than $40 \mathrm{mg}$ per $100 \mathrm{~g}$ of product (Bucchini 2019). Chlorella stigmatophora extract presented $5.27 \mathrm{~g}$ of $\alpha$ linolenic per $100 \mathrm{~g}$ of extract and $1.40 \mathrm{~g}$ of EPA per
$100 \mathrm{~g}$ of extract after hydrolysis, while Hemiselmis andersenii presented $3.85 \mathrm{~g}$ of $\alpha$-linolenic per $100 \mathrm{~g}$ of extract and $4.24 \mathrm{~g}$ of EPA + DHA per $100 \mathrm{~g}$ of extract after hydrolysis.

Although both $\omega 3$ and $\omega 6$ PUFA are essential for human nutrition, a balance between the ingestion of these two series of PUFA is crucial for maintaining health (de Carvalho and Caramujo 2018). The western diet favors the consumption of $\omega 6$ PUFA leading to an unbalanced $\Sigma \omega 3 / \Sigma \omega 6$ ratio of $1: 20$ (Simopoulos 2016). The $\omega 3$ series of fatty acids are known for their anti-inflammatory properties while the $\omega 6$ series are known as precursors of pro-inflammatory molecules (Simopoulos 2016). Thus, an unbalanced diet incorporating great amounts of $\omega 6$ PUFA can have prothrombotic and pro-inflammatory implications (de Carvalho and Caramujo 2018). To achieve a dietary balance of $\Sigma \omega 3 / \Sigma \omega 6$ of $1: 1$, the consumption of $\omega 3$ PUFA-rich sources is recommended to prevent and mitigate inflammatory diseases. In the present study, both microalgae presented a $\Sigma \omega 3 / \Sigma \omega 6>1$, suggesting that they are good sources for supplying a $\omega 3$-PUFA-rich diet for aquaculture and nutraceuticals. The greatest $\Sigma \omega 3 /$ $\Sigma \omega 6$ ratio was verified for Hemiselmis andersenii.

Aliphatic alcohols. C10-C20 aliphatic alcohols with C16-OH abundance were previously reported for 

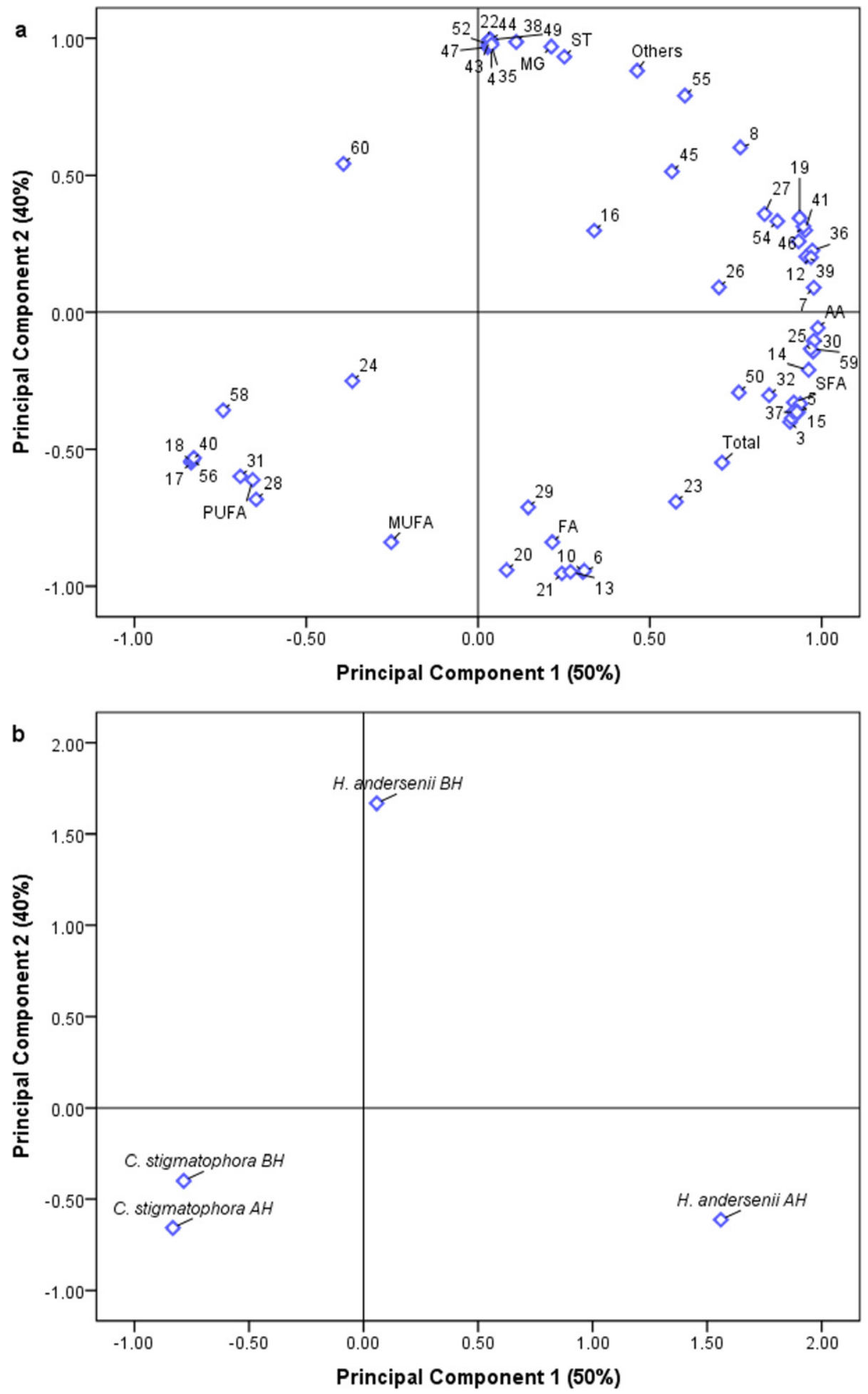

FIG. 5. Projection (Varimax rotation) of the a) loadings and b) scores of components 1 and 2 obtained in the Principal Component Analysis based on the composition of Chlorella stigmatophora and Hemiselmis andersenii lipophilic extracts before (BH) and after (AH) alkaline hydrolysis. The corresponding identification of the numbered loadings is displayed in Table 1. AA, Aliphatic alcohols; FA, fatty acids; SFA, saturated fatty acids; MUFA, monounsaturated fatty acids; PUFA, polyunsaturated fatty acids; MG, monoglycerides; ST, sterols. [Colour figure can be viewed at wileyonlinelibrary.com]

species belonging to Chlorella genus (Chlorella kessleri; Volkman et al., 1998). In C. stigmatophora, the predominant aliphatic alcohols were those with shorter chain lengths (C10-C20). Docosanol (C22-OH) was found in phytoplankton and sediment samples where an algal origin is plausible (Volkman et al., 
1998). This alcohol was only detected in small amounts for Hemiselmis andersenii samples.

Phytol comprises the hydrophobic tail of chlorophylls (Yao et al. 2015, Laurens et al. 2017) which can explain the large contribution of this compound to the hydrolyzed lipid fraction of Chlorella stigmatophora. Phytol is presently used as a fragrance constituent, and it has drawn increasing attention from the pharmaceutical and biotechnological fields due to its abundance in biological systems and diverse bioactivities-including cytotoxic, anti-anxiety and anti-inflammatory effects (Islam et al. 2018).

Previous studies have reported the presence of C28-C32 saturated alkyl diols in cyanobacteria and Eustigmatophytes (Nannochloropsis, Vischeria punctata, Vischeria helvetica, and Eustigmatos vischeri; Volkman et al., 1998). These diols have been suggested to be the building blocks for novel aliphatic biopolymers synthesized by microalgae and cyanobacteria. According to Volkman et al. (1998), there is a strong possibility for long-chain alkyl diols to be found in other algal classes, and this observation is corroborated by our results.

Policosanol is a mixture of LCAA that is commercialized as a lipid-lowering supplement (Cherif 2011). According to Taylor et al. (2003), the dietary intake of $20 \mathrm{mg} \cdot \mathrm{d}^{-1}$ of policosanol is as effective as $100 \mathrm{mg} \cdot \mathrm{d}^{-1}$ of aspirin in the prevention of thrombosis and cerebral ischemia with the advantage of causing no side effects. Few reports address the presence of LCAA in microalgal biomass (Volkman et al. 1992, Gelin 1996). In contrast, Fernandes et al. (2020) find great amounts of LCAA in a Haptophyta microalga, Pavlova pinguis. This finding is in agreement with the LCAA contents found for both Hemiselmis andersenii and Chlorella stigmatophora, where $20 \mathrm{mg}$ of policosanol is equivalent to 9.09 and $5.76 \mathrm{~g}$ of dried biomass, respectively.

Sterols and monoglycerides. Throughout algal classes, there is a predominance of specific sterols. The major sterols in Cryptophyta are C28 $\Delta^{5,22}$, for instance brassicasterol, while in chlorophyta, the prevalent sterols are C28 $\Delta^{5}$ and C29 $\Delta^{5}$ sterols such as campesterol and sitosterol, respectively (Volkman et al. 1998, Taipale et al. 2016). These observations are in accordance with the results obtained for Hemiselmis andersenii and Chlorella stigmatophora. The brassicasterol dominance in the Hemiselmis sterol family is also consistent with that previously reported by Dunstan et al. (2005) for other Cryptophyta species.

Previous studies have highlighted the bioactivities of sterols: brassicasterol is recognized for its antiaging and cholesterol-lowering properties, while campesterol is known for its anticancer and antiangiogenic properties (Luo et al. 2015, Peltomaa et al. 2017). From the detected phytosterols, $\beta$-sitosterol is the most valuable sterol, since it exhibits several bioactive properties like anti-inflammatory, antineoplastic, antipyretic, and immunomodulating activities, and has been subject of FDA health claims (Cherif 2011, Luo et al. 2015).

Monoglycerides are used in the food industry as emulsifiers, stabilizers, and/or thickeners (Wang and Marangoni 2016). In living systems, acylglycerols (mono-, di-, and triacylglycerols) are recognized for their storage role and are often composed of C16, C18, and C20 carboxylic acids (Bianchi and Canuel 2011). In Hemiselmis andersenii, esters of glycerol with saturated C16 and C18 fatty acids were detected. In contrast to acylglycerols, alkylglycerol ether bonds are stable to alkali, explaining the detection of these molecules after hydrolysis in $H$. andersenii (Christie 1989).

Principal component analysis. The sites of cleavage via alkaline hydrolysis (saponification) are the ester bonds of lipids, which result in a carboxylic acid and alcohol that are recovered and derivatized for further GC-MS analysis. This method targets complex lipids-like acylglycerols (mono-, di-, and triacylglycerols), wax, and sterol esters.

The chemical divergence observed in the GC-MSbased metabolite profile of Hemiselmis andersenii before $(\mathrm{BH})$ and after $(\mathrm{AH})$ hydrolysis is mainly explained by the detection of monoacylglycerols and other metabolites with strong positive correlation with PC2, which were only present in metabolite profiles of $H$. andersenii $\mathrm{BH}$. In $H$. andersenii, the concomitant increase of fatty acids with aliphatic alcohols observed after alkaline hydrolysis suggests that wax esters are abundant in this species.

Regarding Chlorella stigmatophora, only fatty acids significantly increased, as well as phytol after hydrolysis. This might be explained by the fact that lipids are mainly constituted by polar lipids (glyco- and phospholipids) and non-acylglycerol neutral lipids like chlorophylls, which are more stable to alkaline hydrolysis. This assumption also explains the similarities in the lipophilic metabolite profiles for C. stigmatophora before and after alkaline hydrolysis observed in PCA analysis.

PCA results showed that Chlorella stigmatophora samples were richer in PUFA, oleic acid, $\beta$-sitosterol, and LA, which suggests that this microalga is a good source of essential fatty acids needed to fulfill the dietary requirements of humans and fish. Hemiselmis andersenii $\mathrm{BH}$ presented higher levels of sterols, monoglycerides, and DHA, and $H$. andersenii $\mathrm{AH}$ had higher amounts of the total identified compounds and SFA. This indicates that $H$. andersenii composition can be suitable not only for nutraceuticals (sterols and DHA) but also for the food industry (monoglycerides) and bioenergy (SFA).

\section{CONCLUSIONS}

Microalgal lipids are often assessed for their biotechnological potential, taking into consideration one specific family, namely fatty acids. This approach constrains microalgae strain selection for 
further industrial applications. Since FTIR-ATR and GC-MS metabolite profile provides an overall insight into microalgae intracellular metabolites, it can be used to identify components that may serve as chemotaxonomical markers as well as novel natural sources of high-value compounds. The present study contributed to the valorization of Chlorella stigmatophora and Hemiselmis andersenii as sources of value-added compounds with recognized nutritional benefits. Although the mere presence of these health-promoting phytochemicals may not support its use in a particular application/formulation, their knowledge provides a novel insight for further studies and final product optimization targeting the field of application.

\section{ACKNOWLEDGEMENTS}

This work was supported by the European Territorial Cooperation Programme PCT-MAC 2014-2020 through project REBECA-CCT (MAC/1.1.B/269). Tomásia Fernandes thanks ARDITI (Regional Agency for Development of Research, Technology, and Innovation of Madeira), Project M1420-095369-FSE-000002, for the doctoral grant.

\section{AUTHORS' CONTRIBUTION}

T. Fernandes and N. Cordeiro contributed to the conception and design of the study. Tomásia Fernandes executed the experiment, analyzed and interpreted the data, and wrote the manuscript. N. Cordeiro made possible the experiment execution with administrative and financial support, supervised the experimental, and made the critical revision for the important intellectual content of the manuscript.

Bianchi, T. S. \& Canuel, E. A. 2011. Chemical Biomarkers in Aquatic Ecosystems. Princeton University Press, UK, 417 pp.

Borowitzka, M. A. 2013. Species and Strain Selection. In Borowitzka, M. A. \& Moheimani, N. R. [Eds.] Developments in Applied Phycology: Algae for Biofuels and Energy. Springer, pp. $77-89$.

Bucchini, L. 2019. Nutrition and health claims in Europe: oils \& fats related claims, regulatory and labeling challenges. $\mathrm{Ocl}$ $26: 48$.

Cherif, A. O. 2011. In Rasooli, I. [Ed.] Bioactive Compounds in Phytomedicine. InTech, Croatia. pp. 113-24.

Christie, W. W. 1989. Gas Chromatography and Lipids: A Practical Guide. Oily Press, Scotland, 307 pp.

Christie, W. W.2018. The LipidWeb. https://www.lipidhome.co. $\mathrm{uk} /$.

da Costa, E., Silva, J., Mendonca, S. H., Abreu, M. H. \& Domingues, M. R. 2016. Lipidomic approaches towards deciphering glycolipids from microalgae as a reservoir of bioactive lipids. Mar. Drugs 14:101.

de Carvalho, C. \& Caramujo, M. J. 2018. The various roles of fatty acids. Molecules 23:2583.

Driver, T., Bajhaiya, A. K., Allwood, J. W., Goodacre, R., Pittman, J. K. \& Dean, A. P. 2015. Metabolic responses of eukaryotic microalgae to environmental stress limit the ability of FT-IR spectroscopy for species identification. Algal Res 11:148-55.

Dunstan, G. A., Brown, M. R. \& Volkman, J. K. 2005. Cryptophyceae and rhodophyceae; chemotaxonomy, phylogeny, and application. Phytochemistry 66:2557-70.
Fernandes, T., Fernandes, I., Andrade, C. A. P. \& Cordeiro, N. 2016. Marine microalgae growth and carbon partitioning as a function of nutrient availability. Bioresour. Technol. 214:541-47.

Fernandes, T., Martel, A. \& Cordeiro, N. 2020. Exploring Pavlova pinguis chemical diversity: a potentially novel source of high value compounds. Sci. Rep. 10:339.

Forfang, K., Zimmermann, B., Kosa, G., Kohler, A. \& Shapaval, V. 2017. FTIR spectroscopy for evaluation and monitoring of lipid extraction efficiency for oleaginous fungi. PLoS ONE 12: e0170611.

Gelin, F.1996. Isolation and chemical characterisation of resistant macromolecular constituents in microalgae and marine sediments. PhD thesis, Utrecht University, $147 \mathrm{pp}$.

Gheysen, L., Bernaerts, T., Bruneel, C., Goiris, K., Van Durme, J., Van Loey, A., De Cooman, L. \& Foubert, I. 2018. Impact of processing on n-3 LC-PUFA in model systems enriched with microalgae. Food Chem. 268:441-50.

Guiheneuf, F. \& Stengel, D. B. 2013. LC-PUFA-enriched oil production by microalgae: accumulation of lipid and triacylglycerols containing n-3 LC-PUFA is triggered by nitrogen limitation and inorganic carbon availability in the marine haptophyte Pavlova lutheri. Mar. Drugs 11:4246-66.

Huerlimann, R., de Nys, R. \& Heimann, K. 2010. Growth, lipid content, productivity, and fatty acid composition of tropical microalgae for scale-up production. Biotechnol. Bioeng. 107:245-57.

Islam, M. T., Ali, E. S., Uddin, S. J., Shaw, S., Islam, M. A., Ahmed, M. I., Chandra Shill, M. et al. 2018. Phytol: a review of biomedical activities. Food Chem. Toxicol. 121:82-94.

Jiang, Q., Zhao, L., Dai, J. \& Wu, Q. 2012. Analysis of autophagy genes in microalgae: Chlorella as a potential model to study mechanism of autophagy. PLoS ONE 7:e41826.

Jones, P. J. \& AbuMweis, S. S. 2009. Phytosterols as functional food ingredients: linkages to cardiovascular disease and cancer. Curr. Opin. Clin. Nutr. Metab. Care 12:147-51.

Kent, M., Welladsen, H. M., Mangott, A. \& Li, Y. 2015. Nutritional evaluation of Australian microalgae as potential human health supplements. PLOS ONE 10:e0118985.

Khannoon, E. R., Flachsbarth, B., El-Gendy, A., Mazik, K., Hardege, J. D. \& Schulz, S. 2011. New compounds, sexual differences, and age-related variations in the femoral gland secretions of the lacertid lizard Acanthodactylus boskianus. Biochem. Syst. Ecol. 39:95-101.

Lane, C. E., van den Heuvel, K., Kozera, C., Curtis, B. A., Parsons, B. J., Bowman, S. \& Archibald, J. M. 2007. Nucleomorph genome of Hemiselmis andersenii reveals complete intron loss and compaction as a driver of protein structure and function. Proc. Natl. Acad. Sci. USA 104:19908-13.

Laurens, L. M. L., Markham, J., Templeton, D. W., Christensen, E. D., Van Wychen, S., Vadelius, E. W., Chen-Glasser, M., Dong, T., Davis, R. \& Pienkos, P. T. 2017. Development of algae biorefinery concepts for biofuels and bioproducts; a perspective on process-compatible products and their impact on cost-reduction. Energy Environ. Sci. 10:1716-38.

Lum, K. K., Kim, J. \& Lei, X. G. 2013. Dual potential of microalgae as a sustainable biofuel feedstock and animal feed. $J$. Anim. Sci. Biotechnol. 4:1-7.

Luo, X., Su, P. \& Zhang, W. 2015. Advances in microalgae-derived phytosterols for functional food and pharmaceutical applications. Mar. Drugs 13:4231-54.

Ma, N. L., Teh, K. Y., Lam, S. S., Kaben, A. M. \& Cha, T. S. 2015. Optimization of cell disruption methods for efficient recovery of bioactive metabolites via NMR of three freshwater microalgae (Chlorophyta). Bioresour. Technol. 190:536-42.

Matich, E. K., Ghafari, M., Camgoz, E., Caliskan, E., Pfeifer, B. A., Haznedaroglu, B. Z. \& Atilla-Gokcumen, G. E. 2018. Timeseries lipidomic analysis of the oleaginous green microalga species Ettlia oleoabundans under nutrient stress. Biotechnol. Biofuels 11:29.

Muys, M., Sui, Y., Schwaiger, B., Lesueur, C., Vandenheuvel, D., Vermeir, P. \& Vlaeminck, S. E. 2019. High variability in nutritional value and safety of commercially available 
Chlorella and Spirulina biomass indicates the need for smart production strategies. Bioresour. Technol. 275:247-57.

Peltomaa, E., Johnson, M. D. \& Taipale, S. J. 2017. Marine cryptophytes are great sources of EPA and DHA. Mar. Drugs 16:3.

Rahmouni, N., Pinto, D. C. G. A., Santos, S. A. O., Beghidja, N. \& Silva, A. M. S. 2017. Lipophilic composition of Scabiosa stellata L.: an underexploited plant from Batna (Algeria). Chem. Pap. 72:753-62.

Rocha, R. P., Machado, M., Vaz, M. G. M. V., Vinson, C. C., Leite, M., Richard, R., Mendes, L. B. B. et al. 2017. Exploring the metabolic and physiological diversity of native microalgal strains (Chlorophyta) isolated from tropical freshwater reservoirs. Algal Res. 28:139-50.

Santos, S. A., Vilela, C., Freire, C. S., Abreu, M. H., Rocha, S. M. \& Silvestre, A. J. 2015. Chlorophyta and Rhodophyta macroalgae: a source of health promoting phytochemicals. Food Chem. 183:122-8.

Simopoulos, A. P. 2016. An increase in the omega-6/omega-3 fatty acid ratio increases the risk for obesity. Nutrients 8:128.

Slocombe, S. P., Zhang, Q., Ross, M., Anderson, A., Thomas, N. J., Lapresa, A., Rad-Menendez, C., Campbell, C. N., Black, K. D., Stanley, M. S. \& Day, J. G. 2015. Unlocking nature's treasure-chest: screening for oleaginous algae. Sci. Rep. 5:9844.

Sukenic, A. 1991. Ecophysiological considerations in the optimization of Eicosapentaenoic acid production by Nannochloropsis sp. (Eustigmatophyceae). Bioresour. Technol. 35:263-69.

Taipale, S. J., Hiltunen, M., Vuorio, K. \& Peltomaa, E. 2016. Suitability of phytosterols alongside fatty acids as chemotaxonomic biomarkers for phytoplankton. Front. Plant Sci. 7:212.

Taylor, J. C., Rapport, L. \& Lockwood, G. B. 2003. Octacosanol in human health. Nutrition 19:192-95.

Volkman, J. K., Barrett, S. M., Blackburn, S. I., Mansour, M. P., Sikes, E. L. \& Gelin, F. 1998. Microalgal biomarkers: a review of recent research developments. Org. Geochem. 29:1163-79.

Volkman, J. K., Barrett, S. M., Dunstan, G. A. \& Jeffrey, S. W. 1992. C30-C32 alkyl diols and unsaturated alcohols in microalgae of the class Eustigmatophyceae. Org. Geochem. 18:131-38.
Wang, F. C. \& Marangoni, A. G. 2016. Advances in the application of food emulsifier alpha-gel phases: Saturated monoglycerides, polyglycerol fatty acid esters, and their derivatives. $J$. Colloid. Interface Sci. 483:394-403.

Xin, L., Hu, H. Y., Ke, G. \& Sun, Y. X. 2010. Effects of different nitrogen and phosphorus concentrations on the growth, nutrient uptake, and lipid accumulation of a freshwater microalga Scenedesmus sp. Bioresour. Technol. 101:5494-500.

Xu, B., Zhang, L., Ma, F., Zhang, W., Wang, X., Zhang, Q., Luo, D., Ma, H. \& Li, P. 2018. Determination of free steroidal compounds in vegetable oils by comprehensive two-dimensional gas chromatography coupled to time-of-flight mass spectrometry. Food Chem. 245:415-25.

Yao, L., Gerde, J. A., Lee, S. L., Wang, T. \& Harrata, K. A. 2015. Microalgae lipid characterization. J. Agric. Food Chem. 63:1773-87.

\section{Supporting Information}

Additional Supporting Information may be found in the online version of this article at the publisher's web site:

Figure S1 Growth curves for Chlorella stigmatophora and Hemiselmis andersenii.

Figure S2 Fourier Transform Infrared spectroscopy with Attenuated Total Reflectance (FTIR-ATR) spectra of freeze-dried microalga (raw microalga) and lipophilic extract before hydrolysis a) Chlorella stigmatophora and b) Hemiselmis andersenii. 